\title{
Benefits on language development and auditory perception performance of using a contralateral hearing aid in cochlear implanted children
}

\author{
Mustafa Colak', İsmet Bayramoğlu², Hakan Tutar², Șenay Altınyay² \\ 1 Ankara City Hospital, Department of Otorhinolaryngology, Ankara, Turkey \\ 2 Gazi University Faculty of Medicine, Department of Otorhinolaryngology, Ankara, Turkey \\ Mustafa Çolak, ORCID: 0000-0002-3191-4134 \\ Ismet Bayramoğlu, ORCID: 0000-0003-4650-8005, \\ Hakan Tutar, ORCID: 0000-0001-5585-5282, \\ Şenay Altınyay, ORCID: 0000-0002-5622-6194
}

\begin{abstract}
Objective: To evaluate the benefits of a contralateral hearing aid on expressive and receptive language development and auditory perception performance over a 36-month post-surgical period in children implanted on a single side with a cochlear device for bilateral pre-lingual profound sensorineural hearing loss.
\end{abstract}

Methods: Seventy-five patients with idiopathic profound sensorineural hearing loss were included. The cases were divided into two groups: cochlear implant users (50 patients, "Cl group") and cochlear implant plus hearing aid users (25 patients, " $\mathrm{Cl}+\mathrm{HA}$ group"). Language development and auditory performance were compared in the two groups during the first 3 years following cochlear implant surgery. The Pre-school Language Scale- 4 was used to assess language development and the LittIEars $₫$ Auditory Survey, Meaningful Auditory Integration Scale and Meaningful Use of Speech Scale were employed to assess auditory perception performance.

Results: Language development in the $\mathrm{Cl}+\mathrm{HA}$ group was superior to that in the $\mathrm{Cl}$ group at 6 months post-surgery, in terms of receptive and expressive language development; auditory perception performance was also superior in the $\mathrm{Cl}+\mathrm{HA}$ group, compared to the $\mathrm{Cl}$ group.

Conclusion: The use of a contralateral hearing aid in cochlear implanted children with prelingual sensorineural hearing loss positively contributes to language devel-opment and auditory perception performance.

Keywords: Auditory perception, cochlear implants, hearing aids, language development. 
Hearing loss is one of the most common disabilities encountered in children. If not corrected, its negative effects may last a lifetime. Sensorineural hearing loss occurs in approximately 1-2 infants in every 1,000 births. ${ }^{[1,2]}$ Hearing loss may be congenital or acquired after birth. Whatever the cause, hearing loss in the pre-lingual period should be diagnosed as soon as possible, and hearing amplification provided.

The first years of life represent the most critical period for speech and language development. If hearing loss in a child goes unrecognised during this period, speech and language development at the desired level cannot be achieved. Children who do not have auditory perception skills have an inadequate ability to recognise, distinguish, and understand voices. Unrecognised hearing loss during infancy and early childhood adversely affects the development of the central nervous system, and social, emotional, cognitive and academic development lags behind compared to the child's healthy peers. ${ }^{[3]}$ Cochlear implants facilitate a significant increase in hearing and speech skills in patients with severe or profound pre-lingual hearing loss when compared to the period preceding implantation of the device. ${ }^{[4]}$ However, unilaterally implanted patients have difficulty locating sounds, such that all sounds appear to be coming either directly from their ear, or to be inside their head. Additionally, their ability to understand speech in every day, noisy or reverberant environments is relatively poor. ${ }^{[5-7]}$

Currently, bilateral cochlear implantation is a common procedure. However, in some patients, cochlear devices cannot be implanted bilaterally due to medical complications, insurance restrictions or personal preference. Combining electrical hearing (i.e. a cochlear device) with an acoustic hearing aid in the other ear may be an option for such patients. This combination of electrical and acoustic stimulation is termed "bimodal stimulation". Binaural hearing via bimodal stimulation can improve speech perception in both quiet and noisy environments, and also improve the ability to localise sounds, determine sound quality, and perceive melodies and other aspects of music. ${ }^{[8,9]}$ Looking at the benefits obtainable, one would be tempted to suppose the presence of residual hearing in the opposite ear were essential. Surprisingly, though, it appears that the benefits of bimodal stimulation are obtainable even where profound hearing loss is present. ${ }^{[10]}$

In our study, we evaluated the effectiveness of providing bimodal hearing (by supplying a contralateral hearing aid alongside a unilateral cochlear implant) on the development of receptive and expressive language, as well as auditory perception performance, in patients with bilateral pre-lingual profound sensorineural hearing loss.

\section{Materials and Methods}

A retrospective case-control study was undertaken at Gazi University Hospital. The study was conducted in accordance with the principles of the Helsinki Declaration. Ethical permission number 469 was granted on 13/10/2014 by the Corporate Ethics Committee of the Gazi University Hospital.

A retrospective review was undertaken of patients from the clinic who had undergone cochlear implantation between 2002 and 2014 for profound pre-lingual sensorineural hearing loss. A total of seventy-five patients (50 single CI users, $25 \mathrm{CI}$ and contralateral HA users) were included in the study. Table 1 presents the demographic data for the study participants. The inclusion criteria were pure-tone averages (PTAs) for hearing thresholds at 0.5, 1, 2 and 4 $\mathrm{kHz}$ worse than $90 \mathrm{~dB}$ hearing loss in both ears, diagnosed in the pre-lingual period. The exclusion criteria were: patients who did not regularly use the cochlear implant or hearing aids, who suffered from hearing loss secondary to meningitis or a specific syndrome, had mental retardation, had not attended the rehabilitation programme regularly or had not attended audiological check-ups on a regular basis. The patients enrolled in the CI+HA group continued to use the hearing aids that they were using before the implant surgery.

In the present study, for the evaluation of language development, a ratio was calculated in which the average score obtained for each language term was compared to the expected score at the patient's chronological age, rather than recording the change over the period in which the patient was assessed. If the value of the ratio obtained is equal to or greater than 1 , the patient's language age is consistent with the chronological age; if it is less than 1, the language age is lower than the chronological age. When the level of language development between groups was compared in our study, this measure was used to compare outcomes.

For each of the participants selected from the audiology service follow-up documentation, the preoperative and postoperative 36-month language development test results and auditory perception test results were entered into a data format suitable for use with Microsoft Excel (Excel Version 12.0, Microsoft, Redmond, WA, USA). Other data, 


\begin{tabular}{|c|c|c|c|}
\hline & $\mathrm{Cl}$ & $\mathrm{Cl}+\mathrm{HA}$ & p \\
\hline Male & $25(50 \%)$ & $9(36 \%)$ & \\
\hline Female & $25(50 \%)$ & $16(64 \%)$ & \\
\hline Diagnosis age* (month) & $9.1 \pm 4.8$ & $10.7 \pm 4.7$ & $p>0.05$ \\
\hline Operation age* (month) & $19.4 \pm 6.6$ & $20 \pm 5.9$ & $\mathrm{p}>0.05$ \\
\hline Duration of hearing aid before surgery* (month) & $5.8 \pm 4.6$ & $6 \pm 4.3$ & $p>0.05$ \\
\hline PTAs $(.5,1,2,4 \mathrm{khz})$ implanted side* (before surgery) (dB) & $104.9 \pm 9.6$ & $104.2 \pm 9.8$ & $\mathrm{p}>0.05$ \\
\hline PTAs $(.5,1,2,4 \mathrm{khz})$ non-operated side* (dB) & $103.5 \pm 9.6$ & $100.2 \pm 9.3$ & $p>0.05$ \\
\hline
\end{tabular}

$\mathrm{Cl}$ : Cochlear implant group, $\mathrm{Cl}+\mathrm{HA}$ : Cochlear implant+hearing aid group, PTAs: pure tone averages, dB: decibel, khz: kilohertz, *: mean \pm SD

including the patients' sex, age of diagnosis, age of operation and pre-operative device usage times were also recorded. We used the Turkish-validated version of the Preschool Language Scale-4 for the assessment of language development. To determine the performance of auditory perception, we used the LittlEARS ${ }^{\circledR}$ Auditory Questionnaire, the Meaningful Auditory Integration Scale (MAIS) and the Meaningful Use of Speech Scale (MUSS).

\section{Tests Used}

\section{Pre-school Language Scale-4 (PLS-4)}

The PLS-4 test, which plays an important role in studies on language development, is a psychometric test to evaluate both the receptive and expressive language skills of children aged up to 6 years and 11 months of age. There are 62 test items for receptive language, 68 test items for expressive language and 104 pages in the illustrated test books. Testing begins at a level at least one year below the chronological age of the patient. For each question, the child's answer format is marked with ' $D$ ', ' $S$ ', or ' $M$ ' (Directly, Spontaneously, Mother or nanny). Each question has a passing criterion stated under the question. A child who meets the passing criterion is awarded 1 point and considered successful for that question. Any child who passes more than half of the questions in each language level then progresses on to the higher level questions. As a result of the test, the development period which the child has reached in terms of receptive or expressive language is determined (9-11 months, 18-23 months, etc). ${ }^{[1]}$

\section{LittIEARS@ Auditory Questionnaire}

The LittlEARS® Auditory Questionnaire is a questionnaire evaluation that shows the auditory development, speech development and pre-verbal speech phase in normal or hearing-impaired children. It evaluates auditory development up to two years of age in hearing children, or in the first two years after a cochlear implant or hearing aid is supplied. It consists of 35 questions marked 'Yes' or 'No'. The 'Yes' option scores 1 point and the 'no' option 0 points. The test has a maximum total score of 35 points. ${ }^{[12]}$

\section{Meaningful Auditory Integration Scale (MAIS)}

This test is a questionnaire consisting of 10 items that can be applied to children at all ages, both before and after implantation. The items evaluate the child's ability to listen with the hearing aid or implant, recognise sounds and combine them with their meaning. The survey is divided into three sections. The first two questions include the initiation of listening; questions three, four, five and six include recognition of voices; the seventh, eighth, ninth and tenth questions concern the ability to ascribe meaning to voices. There are five different response options for each question ( 0 =never, $1=$ rarely, $2=$ sometimes, 3 =frequently, $4=$ always), giving a minimum item score of zero points and a maximum of four points. The test is evaluated over 40 points in total. ${ }^{[13]}$

\section{The Meaningful Use of Speech Scale (MUSS)}

MUSS is used to assess the child's communication strategies, the ability to produce the sounds used in the mother 
tongue, and the ability to control those sounds. The test may be used with children of all ages. The questionnaire may be filled in by parents or teachers. The first three items on the questionnaire assess the patient's voice control, the next five questions examine the speaking voice, and the last two questions evaluate speaking strategies. There are five different response options for each question $(0=$ never, $1=$ rarely, $2=$ sometimes, $3=$ frequently, $4=$ =always), giving a minimum item score of zero points and a maximum of four points. The test is evaluated over 40 points in total. ${ }^{[13]}$

\section{Statistical Analysis}

All data were analysed by means of the Statistical Package for Social Sciences application (SPSS for Windows version 17.0; SPSS Inc., Chicago, IL, USA). The parametric Student's t-test was used to compare language development levels between the groups. Comparisons of within-group language development level over time were made using the paired sample Student's t-test. The non-parametric Mann-Whitney $U$ test was used to compare auditory perception level development between groups, at different monthly intervals. The level set as indicating statistical significance was $\mathrm{p}<0.05$, for all tests.

\section{Results}

There were no statistically significant differences between the $\mathrm{CI}$ and $\mathrm{CI}+\mathrm{HA}$ groups ( $>0.05)$ in mean age at diagnosis, mean time of use of the device before surgery, the age at which surgery was performed, or PTAs before surgery of both implanted ears and non-operated ears.

There was no significant difference in receptive or expressive language development between groups until the 6th month. However, after the sixth month, both the receptive and expressive language development of the $\mathrm{CI}+\mathrm{HA}$ group was significantly greater $(\mathrm{p}<0.05)$. Table 2 and Figure 1 show the mean language development rates of each group. At the end of thirty-six months, the language development of patients in neither group matched chronological age, but the most significant approximation to this goal was in the CI+HA group (Figure 2).

With reference to the results of the MAIS test, there was no difference between the groups up to the 6th month, while the mean test results of the CI+HA were significantly greater for all months from 6 months to 36 months (36th month, $\mathrm{p}=0.01)$. Similarly, the mean MUSS test scores in the CI+HA group were significantly higher after six months, and this significant elevation continued until the 36th month (36th month $\mathrm{p}<0.01$ ). When the LittlEARS ${ }^{\circledR}$ test results were compared, it was observed that the $\mathrm{CI}+\mathrm{HA}$ group received higher scores from the postoperative period up to 12 months, but this result failed to reach the level of statistical significance. The mean scores in the $\mathrm{CI}+\mathrm{HA}$ group were significantly higher from the twelfth to the thirty-six months inclusive. At the end of the thirty-six months, the mean test results of the $\mathrm{CI}+\mathrm{HA}$ group were also significantly higher $(\mathrm{p}=0.01)$. Table 3 and Figure 3 indicate the mean LittlEARS ${ }^{\circ}$, MAIS and MUSS test results for the groups.

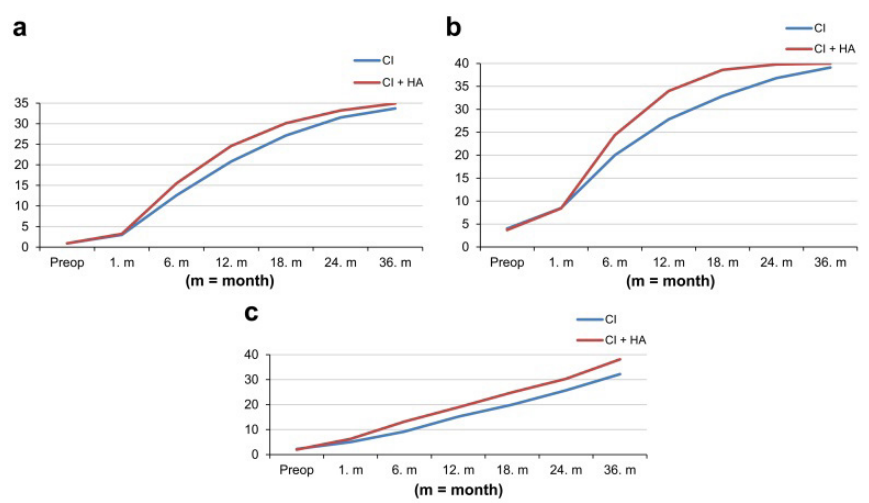

Figure 1. Expressive (a) and Receptive (b) language development of the groups

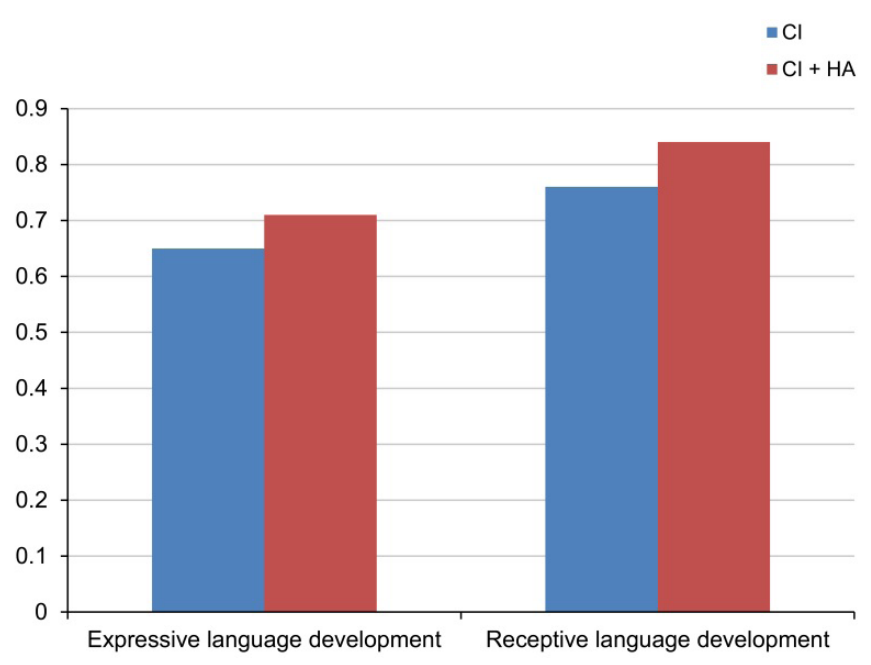

Figure 2. Expressive and receptive language development of the groups at 36th month.

\section{Discussion}

The results of this study suggest that both receptive and 
Table 2. Expressive and receptive language development of $\mathrm{Cl}$ and $\mathrm{Cl}+\mathrm{HA}$ comparison by month

\begin{tabular}{|c|c|c|c|c|c|c|}
\hline & \multicolumn{2}{|c|}{$\mathrm{Cl}$} & \multicolumn{2}{|c|}{$\mathrm{Cl}+\mathrm{HA}$} & \multirow[b]{2}{*}{ P1 } & \multirow[b]{2}{*}{ P2 } \\
\hline & ELD & RLD & ELD & RLD & & \\
\hline 0. $\mathrm{m}^{*}$ & $0.44 \pm 0.13$ & $0.44 \pm 0.12$ & $0.47 \pm 0.12$ & $0.48 \pm 0.13$ & 0.44 & 0.209 \\
\hline 1. $m^{*}$ & $0.43 \pm 0.11$ & $0.44 \pm 0.10$ & $0.47 \pm 0.11$ & $0.50 \pm 0.14$ & 0.11 & 0.032 \\
\hline 6. $m^{*}$ & $0.52 \pm 0.15$ & $0.53 \pm 0.16$ & $0.71 \pm 0.21$ & $0.69 \pm 0.22$ & 0.001 & 0.003 \\
\hline 12. $m$ * & $0.59 \pm 0.20$ & $0.64 \pm 0.19$ & $0.76 \pm 0.17$ & $0.76 \pm 0.16$ & $<0.001$ & 0.011 \\
\hline 18. $m^{*}$ & $0.65 \pm 0.19$ & $0.70 \pm 0.18$ & $0.76 \pm 0.15$ & $0.80 \pm 0.16$ & 0.014 & 0.027 \\
\hline 24. $m^{*}$ & $0.69 \pm 0.20$ & $0.75 \pm 0.18$ & $0.79 \pm 0.16$ & $0.86 \pm 0.16$ & 0.041 & 0.013 \\
\hline 36. $m$ * & $0.65 \pm 0.17$ & $0.71 \pm 0.17$ & $0.76 \pm 0.14$ & $0.84 \pm 0.13$ & 0.009 & 0.002 \\
\hline
\end{tabular}

$\mathrm{Cl}$ : Cochlear implant group, $\mathrm{Cl}+\mathrm{HA}$ : Cochlear implant+Hearing aid group, ELD: expressive language development, m: month, RLD: receptive language development, * mean $\pm \mathrm{SD}$, P1: ELD Cl vs $\mathrm{Cl}+\mathrm{HA}$, P2: RLD Cl vs $\mathrm{Cl}+\mathrm{HA}$
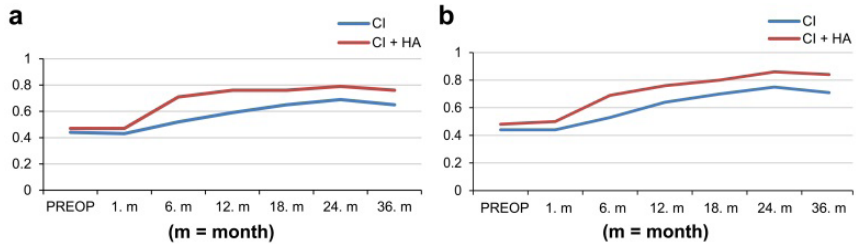

Figure 3. Auditory perception performance of the groups. LittIEARS ${ }^{\circ}(a)$, MAIS (b), MUSS (c) expressive language development and auditory perception performance are significantly better in children using bimodal stimulation hearing aids than in those using a cochlear implant alone. Permanent hearing loss in childhood entails a lifetime of negative impacts on affected individuals and their families. Early diagnosis and treatment of these children are essential for correct speech, language

\begin{tabular}{|c|c|c|c|c|c|c|c|c|c|}
\hline & \multicolumn{3}{|c|}{$\mathrm{Cl}$} & \multicolumn{3}{|c|}{$\mathrm{Cl}+\mathrm{HA}$} & \multirow[b]{2}{*}{ P1 } & \multirow[b]{2}{*}{ P2 } & \multirow[b]{2}{*}{ P3 } \\
\hline & LittIEARS & MAIS & MUSS & LittIEARS & MAIS & MUSS & & & \\
\hline $0 . m^{*}$ & $0.92 \pm 1.3$ & $4 \pm 3.5$ & $2.2 \pm 1.9$ & $0.92 \pm 1.3$ & $3.7 \pm 2.3$ & $2 \pm 1.1$ & $>0.05$ & $>0.05$ & $>0.05$ \\
\hline 1. $m^{*}$ & $3 \pm 2.5$ & $8.5 \pm 4.6$ & $5 \pm 2.9$ & $3.2 \pm 2.1$ & $8.4 \pm 3$ & $6.3 \pm 2.9$ & $>0.05$ & $>0.05$ & $>0.05$ \\
\hline 6. $m^{*}$ & $12.6 \pm 6.2$ & $20 \pm 6.9$ & $9.2 \pm 4.1$ & $15.5 \pm 8.6$ & $24.4 \pm 7.9$ & $13.2 \pm 4.1$ & 0.16 & 0.02 & $<0.01$ \\
\hline 12. $m^{*}$ & $20.8 \pm 6.6$ & $27.8 \pm 6.7$ & $15.2 \pm 5.4$ & $24.6 \pm 6.5$ & $34 \pm 5.1$ & $18.9 \pm 5.1$ & 0.02 & $<0.01$ & $<0.01$ \\
\hline 18. $m$ * & $27.1 \pm 6.1$ & $32.9 \pm 6$ & $20 \pm 6.6$ & $30.1 \pm 4.7$ & $38.6 \pm 2.2$ & $24.9 \pm 4.7$ & 0.03 & $<0.01$ & $<0.01$ \\
\hline 24. $m^{*}$ & $31.5 \pm 4.5$ & $36.8 \pm 1$ & $25.7 \pm 6.7$ & $33.2 \pm 3$ & $39.8 \pm 1$ & $30.3 \pm 3.7$ & 0.02 & $<0.01$ & $<0.01$ \\
\hline 36. $m^{*}$ & $33.7 \pm 3$ & $39.1 \pm 1.9$ & $32.2 \pm 6.5$ & $34.9 \pm 0.2$ & $40 \pm 0$ & $38.1 \pm 3$ & 0.01 & 0.01 & $<0.01$ \\
\hline
\end{tabular}

$\mathrm{Cl}$ : Cochlear implant group, $\mathrm{Cl}+\mathrm{HA}$ : Cochlear implant+hearing aid group, MAIS: Meaningful auditory integration scale, m: month, MUSS: Meaningful use of speech scale, *mean \pm sd P1: LittlEARS $\mathrm{Cl}$ vs $\mathrm{Cl}+\mathrm{HA}$ P2: MAIS Cl vs $\mathrm{Cl}+\mathrm{HA} \mathrm{P3}$ : $\mathrm{MUSS} \mathrm{Cl}$ vs $\mathrm{Cl}+\mathrm{HA}$ 
and cognitive development. Hearing and language learning processes begin at birth and are complete to a greater or lesser extent within approximately four to five years of development in normal children. Neuroplastic activity at this time is at a maximum level, and the various stimuli activate neuronal encoding of language in the fastest and most accurate way. Integration begins within the learning and language centres of the brain. The activity of the auditory cortex begins increasing at birth. ${ }^{[14]}$ This activity of the cortex does not occur in children with bilateral pre-lingual hearing loss of a severe to profound degree. Although unilateral cochlear implants do confer many benefits, including improved speech perceptual ability in implanted children, in daily life, children with cochlear implants continue to experience problems under challenging listening conditions such as noisy classrooms, playgrounds or within the house, due to the auditory stimuli being unilateral. ${ }^{[15]}$ These difficulties can be obviated by bilateral cochlear implants or, where this is not possible, by providing bimodal hearing via the use of a hearing aid.

The benefits of bimodal hearing are reported throughout the literature. ${ }^{[16-18]}$ Speech perception skills when both a cochlear implant and hearing aid are used simultaneously may be better than when hearing is unimodal, using a cochlear implant alone. In patients with bimodal hearing, single-word recognition may undergo a $15 \%-20 \%$ improvement, and a $20 \%-30 \%$ improvement in sentence recognition when exposed to background noise has been observed. Such patients are also better able to locate the origin of sounds. ${ }^{[19-21]}$

There is little doubt that cochlear implants are responsible for improving speech and language skills in children with hearing loss, but their language skills overall continue to be weaker than their hearing peers. In children with hearing loss, bimodal stimulation provides early access to vocal pitch contrasts that are important for early speech perception. ${ }^{[22]}$ Nittrouer and Chapman investigated bilateral cochlear implanted infants with bimodal stimulation experience and found that they manifested improved language development compared to bilaterally implanted infants lacking such experience. ${ }^{[23]}$

The native language of children with CI may exert an effect on language development. Using an identical test battery may introduce a handicap when used to evaluate the development of different languages spoken in different countries. For this reason, different countries should use an appropriately adapted test when assessing development of the particular local language. Iwasaki et al ${ }^{[24]}$ employed a package for the assessment of language development in Japanese hearing-impaired children (ALADJIN) to evaluate the language development in children using cochlear implants and hearing aids together. They report that children who used both a cochlear implant and hearing aid had superior language development to those using a cochlear implant alone. In our study we used the Turkish-language validated version of the Pre-school Language Scale-4. We observed that both expressive and receptive language development were significantly higher in the CI+HA group than in the CI group, from the sixth month following implant surgery.

Perception refers to the ability of our different senses to process the information received from the environment. Auditory perception can be defined as the ability to receive and interpret information reaching our ears through the air or other media in the form of audible frequency waves. Since auditory perception is involved in virtually every task we undertake, naturally it plays a vital role in our daily life, giving us the ability to interact adequately with the environment, communicate fluently, be alerted to potential dangers around us, and even to enjoy music. Numerous studies have proven that unilateral cochlear implantation (i.e. unimodal hearing) enhances auditory perception in children with severe to profound hearing loss. ${ }^{[25,26]}$ Bimodal stimulation is a less well-researched area. Bimodal stimulation promotes central integration of auditory stimuli and supports the acquisition of auditory perceptual skills. Thus, whilst stimulating the contralateral auditory pathway is not crucial for the development of meaningful hearing, it does provide additional perceptual benefits compared to not using the hearing aid. ${ }^{[27]}$ There is evidence suggesting that bimodal stimulation augments the performance of auditory perception by the use of a cochlear implant alone. ${ }^{[28,29]}$ Similarly, in all the three test batteries we used (LittlEars ${ }^{\circledR}$, MAIS and MUSS), we found that auditory perceptual performance was significantly higher in the CI+HA group than in the CI group.

Looking at the benefits obtainable, one would be tempted to suppose the presence of residual hearing in the opposite ear were essential. While electrical stimulation has a greater effectiveness at higher frequencies, acoustic amplification is more effective in boosting the lower frequencies. Bimodal stimulation may be more advantageous than bilateral cochlear implantation in cases where meaningful residual hearing is present, since perception of pitch information, 
including voice pitch contrasts in speech, is directly influenced by the level of hearing at low frequencies. ${ }^{[22,30]}$ Against this explanation, Morera et al ${ }^{[8]}$ state that the amount of residual hearing level cannot be used to predict the benefits of bimodal stimulation. Similarly, Beijen et al ${ }^{[1]}$ hypothesise that the benefits of bimodal stimulation are seen, regardless of whether residual hearing is present or not in the opposite ears of children using unilateral cochlear implants. The results of the present investigation support that hypothesis. Although the PTAs of the patients in the CI+HA group were worse than $100 \mathrm{~dB}$, the results confirmed benefit on language development and auditory perception performance from bimodal stimulation in these patients.

\section{Conclusion}

The key finding in this study is that bimodal stimulation should be recommended to appropriate patients, regardless of the presence of residual hearing, to allow them to experience a social and academic life similar to their hearing peers. Contralateral hearing aid use contributes to language development and auditory perception performance in unilaterally implanted children with profound hearing loss. The retrospective methodology used here imposes certain limitations and, thus, we can expect stronger evidence to be obtainable in future studies using a prospective methodology and enrolling greater numbers of participants.

\section{References}

1. Paludetti G, Conti G, DI Nardo W, et al. Infant hearing loss: from diagnosis to therapy Official Report of XXI Conference of Italian Society of Pediatric Otorhinolaryngology. Acta Otorhinolaryngol Ital 2012;32:34770.

2. Fortnum HM, Summerfield AQ, Marshall DH, Davis AC, Bamford JM. Prevalence of permanent childhood hearing impairment in the United Kingdom and implications for universal neonatal hearing screening: questionnaire based ascertainment study. BMJ 2001;323:536-40.

3. Paradise JL, Dollaghan CA, Campbell TF, et al. Language, Speech Sound Production, and Cognition in Three-Year-Old Children in Relation to Otitis Media in Their First Three Years of Life. Pediatrics 2000;105:111930.

4. Robbins AM, Kirk KI, Osberger MJ, Ertmer D. Speech intelligibility of implanted children. Ann Otol Rhinol Laryngol Suppl 1995;166:399-401.

5. Nelson PB, Jin SH. Factors affecting speech understanding in gated interference: cochlear implant users and normal-hearing listeners. J Acoust Soc Am 2004;115:2286-94.

6. Stickney GS, Zeng FG, Litovsky R, Assmann P. Cochlear implant speech recognition with speech maskers. J Acoust Soc Am 2004;116:1081-91.

7. Stickney GS, Nie K, Zeng FG. Contribution of frequency modulation to
Ethics Committee Approval: The study protocol was approved on 13/10/2014 and permission number 469 was granted by the Gazi University Hospital Corporate Ethics Committee.

Informed Consent: All participants provided written informed consent to the study.

Author Contributions: Designing the study - H.T.; Collecting the data - M.Ç., Ş.A.; Analyzing the data - M.Ç.; Writing the manuscript - M.Ç.; Confirming the accuracy of the data and the analyses - I.B.

Conflict of Interest: The authors have no conflicts of interest to declare.

Financial Disclosure: The authors declare that this study has received no financial support.

speech recognition in noise. J Acoust Soc Am 2005;118:2412-20.

8. Morera C, Manrique M, Ramos A, et al. Advantages of binaural hearing provided through bimodal stimulation via a cochlear implant and a conventional hearing aid: A 6-month comparative study. Acta Otolaryngol 2005;125:596-606.

9. Warren SE, Dunbar MN. Bimodal Hearing in Individuals with Severe-to-Profound Hearing Loss: Benefits, Challenges, and Management. Semin Hear 2018;39:405-13.

10. Ching TY, Psarros C, Hill M, Dillon H, Incerti P. Should children who use cochlear implants wear hearing aids in the opposite ear? Ear Hear 2001;22:365-80.

11. Yalçınkaya F, Bayoglu B, Saraçbaşı O, Belgin E. Turkish adaptation of speech and language disorders test: Preschool Language Scale, fourth edition: PLS-4. Eur J Paediatr Neurol 2007;11:87.

12. Tsiakpini V, Weichbold H, Kuehn-Inacker F, Coninx E, D'Haese P, Almadin S. LittlEARS Auditory Questionnaire. Innsbruck: MED-EL Corp.; 2004.

13. Robbins AM, Renshaw JJ, Berry SW. Evaluating meaningful auditory integration in profoundly hearing-impaired children. Am J Otol 1991; 12:14450. 
14. Gordon KA, Papsin BC, Harrison RV. Activity-dependent developmental plasticity of the auditory brain stem in children who use cochlear implants. Ear Hear 2003;24:485-500.

15. Sarant J, Harris D, Bennet L, Bant S. Bilateral versus unilateral cochlear implants in children: a study of spoken language outcomes. Ear Hear 2014;35:396-409.

16. Litovsky RY, Johnstone PM, Godar SP. Benefits of bilateral cochlear implants and/or hearing aids in children. Int J Audiol 2006;45:78-91.

17. Dunn CC, Tyler RS, Witt SA. Benefit of wearing a hearing aid on the unimplanted ear in adult users of a cochlear implant. J Speech Lang Hear Res 2005;48:668-80.

18. Luntz M, Shpak T, Weiss H. Binaural-bimodal hearing: concomitant use of a unilateral cochlear implant and a contralateral hearing aid. Acta Otolaryngol 2005;125:863-9.

19. Dorman MF, Gifford RH, Spahr AJ, McKarns SA. The benefits of combining acoustic and electric stimulation for the recognition of speech, voice and melodies. Audiol Neurotol 2008;13:105-12.

20. Gifford RH, Dorman MF, McKarns SA, Spahr AJ. Combined electric and contralateral acoustic hearing: word and sentence recognition with bimodal hearing. J Speech Lang Hear Res 2007;50:835-43.

21. Zhang T, Spahr AJ, Dorman MF, Saoji A. Relationship between auditory function of nonimplanted ears and bimodal benefit. Ear Hear 2013;34:133-41.

22. Ching TY, Day J, Van Buynder P, et al. Language and speech perception of young children with bimodal fitting or bilateral cochlear implants. Cochlear Implants Int 2014;15:43-6.

23. Nittrouer S, Chapman C. The Effects of Bilateral Electric and Bimodal
Electric-Acoustic Stimulation on Language Development. Trends Amplif 2009;13:190-205.

24. Iwasaki S, Nishio S, Moteki H, et al. Language development in Japanese children who receive cochlear implant and/or hearing aid. Int J Pediatr Otorhinolaryngol 2012;76:433-8.

25. Allum JH, Greisiger R, Straubhaar S, Carpenter MG. Auditory perception and speech identification in children with cochlear implants tested with the EARS protocol. Br J Audiol 2000;34:293-303.

26. McConkey Robbins A, Koch DB, Osberger MJ, Zimmerman-Phillips S, Kishon-Rabin L. Effect of age at cochlear implantation on auditory skill development in infants and toddlers. Arch Otolaryngol Head Neck Surg. 2004;130:570-4.

27. Di Nardo W, Giannantonio S, Di Giuda D, De Corso E, Schinaia L, Paludetti G. Role of auditory brain function assessment by SPECT in cochlear implant side selection. Acta Otorhinolaryngol Ital 2013;33:23-8.

28. Marsella P, Giannantonio S, Scorpecci A, Pianesi F, Micardi M, Resca A. Role of bimodal stimulation for auditory-perceptual skills development in children with a unilateral cochlear implant. Acta Otorhinolaryngol Ital 2015;35:442-8.

29. De Raeve L, Vermeulen A, Snik A. Verbal cognition in deaf children using cochlear implants: Effect of unilateral and bilateral stimulation. Audiol Neurootol 2015;20:261-6.

30. Ching TY, van Wanrooy E, Dillon H. Binaural-bimodal fitting or bilateral implantation for managing severe to profound deafness: a review. Trends Amplif 2007;11:161-92

31. Beijen JW, Mylanus EA, Leeuw AR, Snik AF. Should a hearing aid in the contralateral ear be recommended for children with a unilateral cochlear implant? Ann Otol Rhinol Laryngol 2008;117:397-403. 